\title{
Ruptured infected pseudoaneurysm of the thoracic aorta
}

\author{
Mohamad Anas Hussain MD, Graham Roche-Nagle MD
}

Competing interests: None declared

This article has been peer reviewed.

The authors have obtained patient consent.

Affiliation: Toronto General Hospital,

University Health Network, Toronto, Ont.

Correspondence to: Mohamad Anas Hussain, mohamad.hussain @ medportal.ca

CMAJ 2014. DOI:10.1503 /cmaj.140315
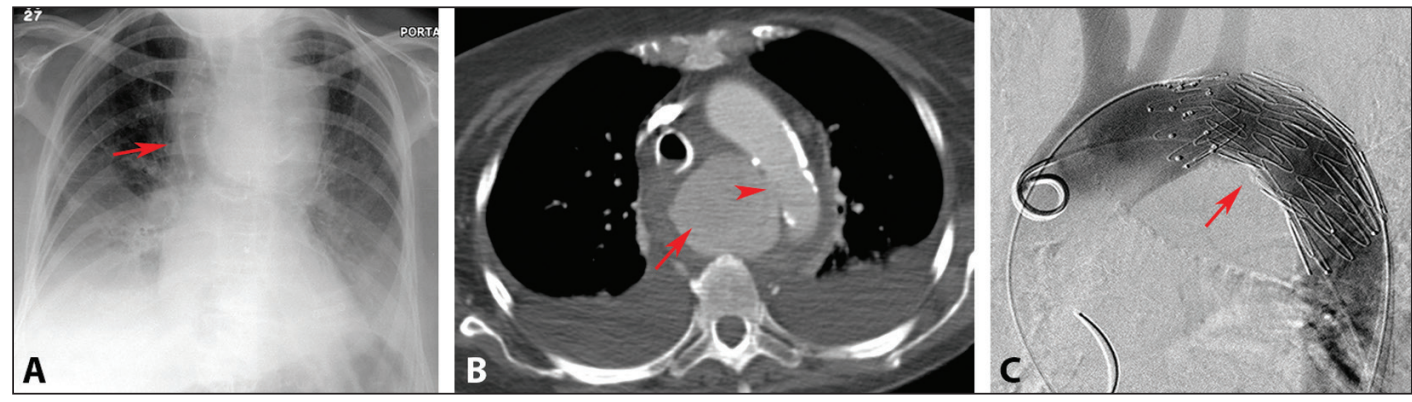

Figure 1: (A) Chest radiograph showing a widened mediastinum and tracheal deviation (arrow) in an 86-year-old woman. (B) Computed tomography showed a pseudoaneurysm of the thoracic aorta (arrow), with a rupture distal to the left subclavian artery (arrowhead). (C) Stent graft (arrow) after endovascular repair is seen on angiogram.

A n 86-year-old woman was admitted to hospital with back pain and general malaise after a fall. Her medical history included hypertension, dyslipidemia and previous venous thromboembolism. Because an initial radiograph of her lumbosacral spine was indeterminate, magnetic resonance imaging was performed and showed lumbar spine discitis. Chest radiography was not done, because she had no respiratory symptoms. Blood cultures grew methicillin-sensitive Staphylococcus aureus, and she was given cefazolin.

Ten days after admission, progressive dyspnea, dysphagia, bilateral swelling of the arms and leukocytosis developed. Chest radiography showed a widened mediastinum, bilateral pleural effusions and tracheal deviation (Figure 1A). Computed tomography of the chest with intravenous contrast showed a saccular thoracic aortic aneurysm $(5.0 \times$ $5.0 \mathrm{~cm}$ ) with surrounding hematoma compressing the trachea, esophagus and superior vena cava (Figure 1B). In the context of bacteremia and discitis, this finding suggested a ruptured infected thoracic pseudoaneurysm. After receiving an endovascular stent graft (Figure 1C) and antibiotic therapy, the patient made a steady recovery and was discharged from hospital 12 days after surgery. At three months' follow-up, her dysphagia and swelling had resolved, but she did have ongoing hoarseness, which was likely caused by direct thoracic aortic pressure on the left recurrent laryngeal nerve (Ortner syndrome).

Infected or mycotic aneurysms of the thoracic aorta are uncommon. They are associated with rapid aortic expansion, rupture and death unless diagnosed early. Diagnosis is based on radiological findings of a nonatherosclerotic saccular aneurysm associated with bacterial sepsis and back or abdominal pain. Before the antibiotic era, the most common cause for mycotic aneurysm was bacterial endocarditis secondary to enterococcal, streptococcal or pneumococcal infection. ${ }^{1}$ As the use of antibiotics, immunosuppression and the use of intravenous drugs have increased, $S$. aureus and Salmonella species have become the predominant organisms. ${ }^{2}$

The gold standard for treatment is open surgical resection of the infected aorta, wide débridement of tissues and reconstruction to re-establish blood flow, but this approach is associated with high mortality (up to $40 \%$ ). ${ }^{3}$ Less invasive endovascular aortic repair shows a mortality benefit. ${ }^{3}$ This treatment is generally used as a bridge for patients who are clinically stable, because stent placement without débridement presents an ongoing risk for infection. Our patient's age made her a poor candidate for surgery; her definitive treatment was limited to endovascular repair and chronic suppressive antibiotics.

\section{References}

1. Brown SL, Busuttil RW, Baker JD, et al. Bacteriologic and surgical determinants of survival in patients with mycotic aneurysms. J Vasc Surg 1984;1:541-7.

2. Malouf JF, Chandrasekaran K, Orszulak TA. Mycotic aneurysms of the thoracic aorta: a diagnostic challenge. Am J Med 2003; 115:489-96.

3. Vallejo N, Picardo NE, Bourke P. The changing management of primary mycotic aortic aneurysms. J Vasc Surg 2011;54:334-40. 